Published in "Acta Astronautica 68(9-10): 1589-1594, 2011"

which should be cited to refer to this work.

\title{
Does mental arithmetic before head up tilt have an effect on the orthostatic cardiovascular and hormonal responses?
}

\author{
Nandu Goswami ${ }^{\mathrm{a}, *}$, Helmut Karl Lackner ${ }^{\mathrm{b}}$, Ilona Papousek ${ }^{\mathrm{c}}$, Jean-Pierre Montani ${ }^{\mathrm{d}}$, \\ Daniela Jezova ${ }^{\mathrm{e}}$, Helmut G. Hinghofer-Szalkay ${ }^{\mathrm{a}, \mathrm{b}}$ \\ a Institute of Physiology, Center of Physiological Medicine, Medical University Graz, 8010 Graz, Austria \\ ${ }^{\mathrm{b}}$ Institute of Adaptive and Spaceflight Physiology, Wormgasse 9, Graz, Austria

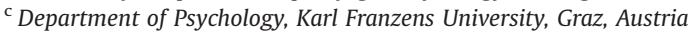 \\ d Department of Medicine/Physiology, University of Fribourg, Switzerland \\ e Institute of Experimental Physiology, Slovak Academy of Sciences, Bratislava, Slovakia
}

\begin{abstract}
Passive head up tilt (HUT) and mental arithmetic (MA) are commonly used for providing mental and orthostatic challenges, respectively. In animal experiments, even a single exposure to a stressor has been shown to modify the response to subsequent stress stimulus. We investigated whether MA applied before HUT elicits synergistic responses in orthostatic heart rate (HR), cardiac output (CO), heart rate variability and arterial blood pressure. The 15 healthy young males were subjected to two randomized protocols: (a) HUT and (b) HUT preceded by MA, with sessions randomized and $\geq 2$ weeks apart. Beat to beat continuous hemodynamic variables were measured and saliva samples taken for hormonal assay. HUT alone increased HR from $59 \pm 7$ (baseline) to $80 \pm 10 \mathrm{bpm}$ (mean \pm SD) and mean blood pressure (MBP) from $88 \pm 10$ to $91 \pm 14 \mathrm{mmHg}$. HUT results after MA were not different from those with HUT alone. The activity of alpha amylase showed differences during the experiments irrespective of the protocols. We conclude that mental challenge does not affect orthostatic cardiovascular responses when applied before; the timing of mental loading seems to be critical if it is intended to alter cardiovascular responses to upright standing.
\end{abstract}

\section{Introduction}

A common physical stress to the human body is standing, which may lead to dizziness in normal persons. When a healthy person stands, there is $10-15 \%$ (approximately $600-700 \mathrm{ml}$ ) of the blood being redistributed to the legs. This leads to decreases in venous return (cardiac pre-load), cardiac filling pressure and output. With normal regulatory capability, arterial pressure at heart level remains unaltered or even is slightly increased. Passive head up tilt (HUT) is used regularly as an orthostatic loading tool.

\footnotetext{
* Corresponding author. Tel.: +433163804278.

E-mail address: Nandu.goswami@meduni-graz.at (N. Goswami).
}

The neurovascular responses to mental stress can take varied shapes: Mental challenge activates the sympathetic system, increases heart rate, cardiac output and blood pressure [1]. Mental arithmetic (MA) is applied routinely to provide mental challenge.

While central drive induced by mental challenge adds to physiologically mediated cardiovascular reflexes and affects orthostatic responses [30], are these changes present when additional mental loading is applied before orthostatic challenge? From animal experiments, it has been shown that even a single exposure to a stressor may modify the response to a subsequent stress stimulus $[2,3]$. For example, repeated exposure to some but not all stress stimuli may lead to a desensitization of responses to the primary persistent stress, but hyperresponsiveness to a novel stimulus $[4,5]$. We, therefore, hypothesized that 
mental challenge preceding HUT would also affect the orthostatic cardiovascular responses. This would have practical value since if this is right, it would imply that mental challenge could be applied in subjects even before they stand up, and the resulting cortical activation would improve the orthostatic cardiovascular responses upon standing up. We investigated this using HUT alone and HUT preceded by MA. Stress application was randomized and separated by two weeks.

\section{Materials and methods}

\subsection{Participants}

The study was carried out with healthy, non-obese, non-smoking, non-medicated men who were free from any somatic or mental condition. Because gender and age may affect orthostatic and stress responses (reviewed in [6]), we focused on young healthy men whose physical characteristics were homogeneous. The study criteria were met by 15 subjects of age $27 \pm 5$ years, weight $74 \pm 8 \mathrm{~kg}$ and height $179 \pm 5 \mathrm{~cm}$ (mean \pm SD).

Subjects were familiarized with the test protocol and gave written informed consent to participate in the study. The study was approved by the Graz University Ethics Board and was performed in accordance with the 1989 WMA Declaration of Helsinki.

\subsection{Study design}

The subjects served as their own controls. An online randomizer (http://www.randomizer.org/) was used to allocate the subjects to each protocol. We asked participants to abstain from coffee for 2 days before the test sessions. Since there is an influence of salt intake on baroreceptor sensitivity and because the person's volume status influences hormonal basal levels as well as responses to orthostatic stress [7], test persons were asked not to change their fluid and salt intake as governed by their usual dietary habits.
To compensate for random and unavoidable climatic effects on the cardiovascular system, every day two subjects did either of the two protocols (at 9-11 AM; 11 AM-1 PM). The two protocols were randomized, open and separated by two weeks (Fig. 1): $10 \mathrm{~min}$ of HUT and $10 \mathrm{~min}$ of MA in supine position followed immediately by $10 \mathrm{~min}$ of HUT (HUT preceded by MA). Part of the HUT alone data has been used in the study of Goswami et al. [30] to investigate interaction of mental and orthostatic challenges.

To remove any effects of intravenous cannulation on physiological responses [8], no invasive procedure was done. Saliva was sampled for cortisol and alpha amylase.

\subsubsection{Head up tilt (HUT)}

Each experiment was preceded by 30 min supine rest. At minute zero, the tilt table was brought to $70^{\circ} \mathrm{HUT}$ position and after $10 \mathrm{~min}$, table was returned to supine position (Fig. 1). During the test, subjects were supported by an adjustable footrest, and were instructed to avoid undue movements of the lower limbs and to breathe normally. Test persons were secured and had access to an emergency shutdown (automatic return to supine position) at all times.

Since the aim of the experiment was to induce orthostatic stress without inducing syncope, criteria for termination included any of the following [9]: (a) blood pressure fell below systolic $80 \mathrm{mmHg}$, or that it dropped rapidly (systolic (SBP) by $\geq 20 \mathrm{mmHg} / \mathrm{min}$, diastolic (DBP) by $\geq 10 \mathrm{mmHg} / \mathrm{min}$ ), or heart rate dropped by $\geq 15 \mathrm{bpm}$; (b) Lightheadedness, dizziness, visual disturbances, nausea, stomach awareness, clammy skin, excessive sweating, or skin pallor. These were the criteria of termination but all the subjects went through all the protocols with no problems.

The test was carried out in a semi-dark and quiet room, maintained at $24{ }^{\circ} \mathrm{C}$ and humidity at 55\%, using the Automatized Human Multi-Stimulation Test Device [9].

\subsubsection{Mental arithmetic}

MA was administered by a method similar to that used by Carter and colleagues [1]. Subjects subtracted continuously the numbers 6 or 7, randomly, from a 2 or 3 digit

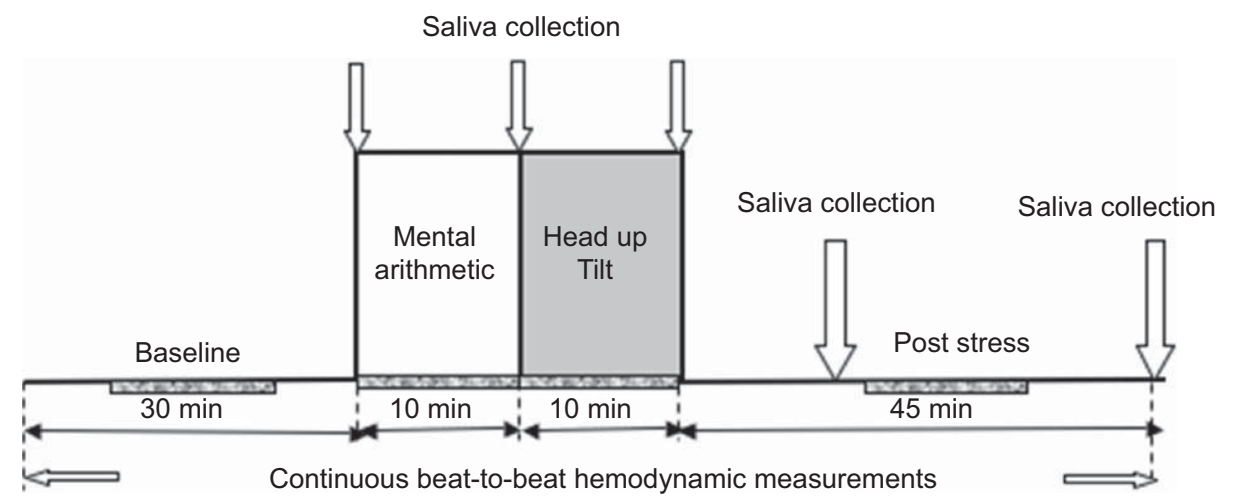

Fig. 1. MA was applied before HUT. Saliva collection was done (a) prior to MA and HUT (sample 1), (b) after finishing MA and/or HUT (sample 2) and at 15 (sample 3) and $45 \mathrm{~min}$ (sample 4) after termination of challenges. Rectangular hatched areas represent durations (10 min each) during which data were analyzed. 
number. A new number was provided every $5 \mathrm{~s}$ to subtract from on a computer screen fixed at the level of the eye of the test subjects.

During the first visit, subjects were familiarized with the laboratory, personnel and equipment. They received standardized verbal instructions about the protocol, tasks, and computer administered MA at the beginning of the first session. Participants were told to solve the tasks as accurately and as fast as possible and that their answers were recorded. Additional pressure was applied by a timer: each calculation lasted only $5 \mathrm{~s}$. Halfway through the MA, subjects were asked to answer more correctly, irrespective of the number of correct answers. These procedures were designed to help reduce adaptation to the stress condition.

\subsection{Self reported measures}

Emotional status was assessed on arrival at the laboratory using the state-trait anxiety inventory (STAI) [10] and the General Depression scale (ADS) [11].

Performance (mistakes made) on the task was assessed and ratings of perception of stress (PSS), shortly before commencing the stresses and retrospectively at the end of mental and/orthostatic challenge, was done using a 5 point Likert scale (1: not stressful; 5 : very, very stressful) [1].

\subsection{Recording physiological stress responses}

Baseline data were collected for $30 \mathrm{~min}$ with the subjects in supine position. During baseline, the subjects were requested to relax without falling asleep. Post-stress the physiological data were obtained for $45 \mathrm{~min}$.

\subsubsection{Hemodynamic monitoring}

Hemodynamic monitoring included blood pressure (upper arm oscillometry and finger plethysmography), heart rate (HR)(3-lead ECG), and thoracic impedance measurements using a Task Force Monitor ${ }^{\circledR}$ (TFM, CNSystems, Graz, Austria), which allows for real-time computation of stroke volume (SV) and total peripheral resistance (TPR). TFM ${ }^{\circledR}$ ECG/impedance electrodes were positioned at the neck and thoracic regions, the latter at the midclavicular line at the xiphoid process level [12]. TPR was calculated as mean arterial blood pressure/ cardiac output and mean arterial pressure from (SBPDBP)/3)+DBP. Pulse pressure (PP) was the difference between systolic (SBP) and diastolic blood pressures (DBP). Impedance cardiography was performed based on the Kubicek approach but using an improved estimate of thoracic volume [12].

\subsubsection{Heart rate and blood pressure variability}

Power spectrum analysis of HR variability assessed sympathovagal balance [13]. Low (LF: $0.04-0.15 \mathrm{~Hz}$ ) and high frequency (HF: $0.15-0.40 \mathrm{~Hz}$ ) power components of RRintervals (RRI), diastolic blood pressure (DBP) and systolic blood pressure (SBP) were evaluated: HFrri (HF-RRV) is primarily mediated by parasympathetic modulation whereas LFrri (LF-RRV) is affected by both parasympathetic and sympathetic activity [14]. Data reported are in normalized units (nu) as the effects appear more consistent when expressed in normalized units [15]. Furthermore, R-R interval variance is often reduced by stressful stimuli [15]. We also calculated LF/HF ratio (indicating sympathovagal balance, (VQ)).

Baroreceptor sensitivity was calculated from the continuous beat-to-beat monitoring of HR and SBP. An increase or a decrease of beat-to-beat RR-interval and SBP in the same direction for at least three cardiac cycles was used to describe the steepness of the regression line [12].

\subsubsection{End tidal $\mathrm{CO}_{2}$ and respiratory frequency}

Because respiratory activity affects arterial $P_{\mathrm{CO}_{2}}, P_{\mathrm{O}_{2}}$ and $\mathrm{pH}$, and modulates sympathetic and vagal efferents [16], end tidal $\mathrm{CO}_{2}$ and respiratory frequency were monitored using a facemask and a capnograph.

\subsection{Saliva}

Salivary cortisol and alpha amylase changes reflect neuroendocrine and autonomic activity [17]. Additionally, alpha amylase in the saliva correlates with plasma norepinephrine in physiological stress paradigms such as in Trier Social stress test, which includes mental arithmetic and public speech [17].

Saliva collection was after $30 \mathrm{~min}$ of baseline, completion of HUT, and/or MA, and at 15 - and 45 min post stress (Fig. 1), using the Salivette ${ }^{\circledR}$ system. Samples were frozen to $-30^{\circ}$ and measured using standard reagent kits not later than one month after sampling.

\section{Analysis of data}

Using typical cardiovascular changes during orthostatic loading from previous studies [18-20], error probability $(\alpha)$ of 0.05 , power $(1-\beta)$ of 0.80 and considering an average effect size (d) of 0.5 , we estimated number of subjects required to be 15 .

All calculations were made with Matlab R2007 (The MathWorks Inc.) and SPSS version 16. HUT only protocol lasted $85 \mathrm{~min}$ : data were analyzed in $10 \mathrm{~min}$ frames representing baseline, stress application and the post stress period (15-25, 30-40 and 60-70 min, respectively). As the MA preceding HUT protocol lasted $95 \mathrm{~min}$, data were also analyzed in 10 min frames representing baseline, MA application, HUT application and post-stress periods (15-25, 30-40, 40-50 and 70-80 min, respectively).

To evaluate the effects of MA on the cardiovascular responses to HUT, $3 \times 2$ multivariate analyses of variance (ANOVAs) were conducted, with phase (baseline, HUT, post stress) and protocol (HUT, HUT preceded by MA) as within-subjects factors, and the cardiovascular measures as the dependent variables. Separate analyses were conducted for selected hemodynamic variables (HR, SV, $\mathrm{CO}$ ), variabilities (HR variability, sympathovagal balance (VQ), baroreceptor sensitivity), blood pressure variables (SBP, DBP, MBP and PP) and vascular resistance (TPR), 
respectively. Two 4 (phase: baseline, HUT, 15 min post stress, $45 \mathrm{~min}$ post stress; within-subject factor) $\times 2$ (protocol; within-subject factor) ANOVAs were performed to analyze effects on alpha-amylase and cortisol responses, respectively.

To compare the hemodynamic, autonomic, salivary hormonal responses resulting due to the two protocols/ type of stress (HUT, HUT preceded by MA) and to assess effects of different phases/condition (baseline, stress, post stress) we used multivariate ANOVA (Fig. 2).

A logarithmic transformation was applied to the sympathovagal balance (VQ) data, as their distribution was skewed.

Paired two-sided Wilcoxon signed rank test was used for comparing emotional stress (ADS, STAI) between the two protocols and to analyze the perception of stress (PSS) within different phases of HUT. Similarly, differences in

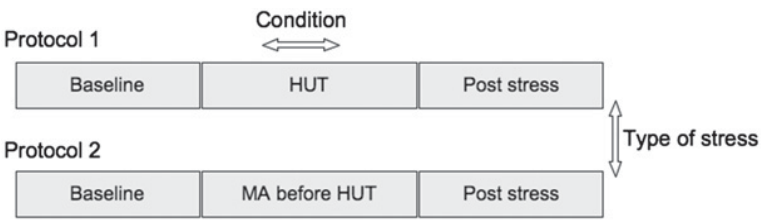

Fig. 2. Summary of the statistical analyses used in this study.
PSS between during- and beginning of HUT and HUT preceded by MA were compared.

\section{Results}

Means and SD of hemodynamic variables and autonomic function are shown in Table 1.

HUT increased HR $(+21 \pm 7 \mathrm{bpm}), \quad \mathrm{MBP}(+3.5 \pm 10$ $\mathrm{mmHg})$ and TPR $\left(+153 \pm 226\right.$ dyne $\left.* \mathrm{~s} / \mathrm{cm}^{5}\right)$ and decreased PP $(-8.6 \pm 7.4 \mathrm{mmHg}), \quad$ SV $(-33.9 \pm 13.5 \mathrm{ml})$ and CO $(-0.6 \pm 1.0 \mathrm{l} / \mathrm{min})$. With the HUT preceded by MA the numbers were HR $(+20.5 \pm 9 \mathrm{bpm})$, MBP $(+7.1 \pm 13.8$ $\mathrm{mmHg})$, TPR $\left(+164 \pm 259\right.$ dyne $\left.* \mathrm{~s} / \mathrm{cm}^{5}\right)$, PP $(-10.2 \pm 9.4$ $\mathrm{mmHg}), \mathrm{SV}(-30.3 \pm 16.6 \mathrm{ml})$ and $\mathrm{CO}(-0.5 \pm 1.3 \mathrm{l} / \mathrm{min})$.

Significant main effects of phase on all cardiovascular variables (HR, SV and CO: $F(6,9)=39.2, p<0.001$; heart rate variabilites and baroreceptor sensitivity: $F(6,9)=13.5$, $p<0.001$; SBP, DBP, MBP and PP: $F(8,7)=8.7, p<0.01$; and TPR: $F(2,13)=11.7, p<0.01$ were seen. The main effects of protocol and the interaction phase $\mathrm{x}$ protocol were not significant for these variables.

Significant main effects of phase were seen on end tidal $\mathrm{CO}_{2}$ and respiratory frequency $(F(4,6)=10.3, p<0.01)$ (Table 1). The main effects of protocol $(F(2,8)=0.9$, ns) and the interaction phase $\mathrm{x}$ protocol were not significant for these variables $(F(4,6)=1.1$, ns).

Table 1

Hemodynamic variables (mean \pm SD) of subjects across Table 1 Acta.xls the two protocols.

\begin{tabular}{|c|c|c|c|c|c|c|}
\hline & \multicolumn{3}{|c|}{ Orthostatic challenge only (HUT) } & \multicolumn{3}{|c|}{ HUT preceded by MA } \\
\hline & Baseline & HUT & Post stress & Baseline & HUT & Post stress \\
\hline Heart rate (bpm) & $59.1 \pm 7.4$ & $80.1 \pm 10.4$ & $57.4 \pm 7.3$ & $63.4 \pm 9.3$ & $83.9 \pm 9.8$ & $61.1 \pm 7.2$ \\
\hline Stroke volume (ml) & $104.7 \pm 16.4$ & $70.9 \pm 9.1$ & $101.7 \pm 16.6$ & $100.7 \pm 17.0$ & $70.4 \pm 8.5$ & $99.7 \pm 15.1$ \\
\hline $\mathrm{CO}(1 / \mathrm{min})$ & $6.2 \pm 1.3$ & $5.6 \pm 0.8$ & $5.8 \pm 1.2$ & $6.3 \pm 1.2$ & $5.9 \pm 0.7$ & $6.1 \pm 1.1$ \\
\hline LFnurri (-) & $56.1 \pm 10.2$ & $77.6 \pm 9.9$ & $54.1 \pm 12.6$ & $54.5 \pm 12.3$ & $79.6 \pm 9.7$ & $56.8 \pm 13.2$ \\
\hline HFnurri (-) & $43.9 \pm 10.2$ & $22.4 \pm 9.9$ & $45.9 \pm 12.6$ & $45.5 \pm 12.3$ & $20.4 \pm 9.7$ & $43.2 \pm 13.2$ \\
\hline Vqrri $(-)$ & $0.29 \pm 0.44$ & $1.46 \pm 0.69$ & $0.20 \pm 0.54$ & $0.23 \pm 0.53$ & $1.48 \pm 0.58$ & $0.20 \pm 0.54$ \\
\hline BRSslope (ms/mmHg) & $27.2 \pm 13.4$ & $9.4 \pm 4.4$ & $29.5 \pm 14.3$ & $26.1 \pm 14.2$ & $9.0 \pm 3.2$ & $28.1 \pm 13.8$ \\
\hline SBP (mmHg) & $120.3 \pm 10.6$ & $116.8 \pm 13.1$ & $116.1 \pm 11.6$ & $116.6 \pm 14.8$ & $116.7 \pm 18.4$ & $118.3 \pm 8.3$ \\
\hline $\mathrm{MBP}(\mathrm{mmHg})$ & $87.7 \pm 10.0$ & $91.2 \pm 13.7$ & $87.2 \pm 10.1$ & $84.9 \pm 13.4$ & $92.0 \pm 15.8$ & $88.8 \pm 7.4$ \\
\hline $\mathrm{DBP}(\mathrm{mmHg})$ & $72.4 \pm 9.8$ & $77.7 \pm 12.8$ & $73.2 \pm 9.4$ & $70.7 \pm 12.2$ & $80.9 \pm 16.2$ & $75.8 \pm 7.5$ \\
\hline PP (mmHg) & $48.7 \pm 8.3$ & $39.2 \pm 7.3$ & $42.8 \pm 6.2$ & $45.8 \pm 7.5$ & $35.6 \pm 9.7$ & $42.5 \pm 6.2$ \\
\hline TPR (dyne $* \mathrm{~s} / \mathrm{cm}^{5}$ ) & $1141 \pm 270$ & $1294 \pm 326$ & $1200 \pm 273$ & $1059 \pm 212$ & $1223 \pm 222$ & $1154 \pm 154$ \\
\hline $\mathrm{ETCO}_{2}(\mathrm{mmHg})$ & $43.0 \pm 2.3$ & $37.8 \pm 1.3$ & $42.2 \pm 1.8$ & $42.9 \pm 1.8$ & $39.2 \pm 3.6$ & $43.3 \pm 2.2$ \\
\hline Respiration (C/min) & $17.0 \pm 2.6$ & $14.4 \pm 3.1$ & $15.9 \pm 3.5$ & $16.6 \pm 3.2$ & $14.2 \pm 2.4$ & $16.1 \pm 4.6$ \\
\hline
\end{tabular}

CO: cardiac output; LFnurri: low frequency $(0.04-0.15 \mathrm{~Hz})$ normalized units; HFnurri: high frequency $(0.15-0.40 \mathrm{~Hz})$ normalized units; VQrri (sympathovagal balance); BRS slope: slope of baroreceptor sensitivity; SBP: systolic blood pressure; MBP: mean blood pressure; DBP: diastolic blood pressure; PP: pulse pressure; TPR: total peripheral resistance; $\mathrm{ETCO}_{2}$ : end tidal carbon dioxide; Respiration: respiratory frequency.

Table 2

Salivary alpha amylase and cortisol responses during HUT and HUT preceded by MA, respectively (mean \pm SD).

\begin{tabular}{|c|c|c|c|c|c|c|c|c|}
\hline & \multicolumn{4}{|c|}{ Orthostatic challenge only (HUT) } & \multicolumn{4}{|c|}{ HUT preceded by MA } \\
\hline & Baseline & HUT & $15^{\prime}$ post stress & $45^{\prime}$ post stress & Baseline & HUT & $15^{\prime}$ post stress & $45^{\prime}$ post stress \\
\hline Alpha-amylase (U/ml) & $24.6 \pm 15.9$ & $27.1 \pm 22.1$ & $24.8 \pm 19.4$ & $40.0 \pm 32.8$ & $29.3 \pm 21.8$ & $29.1 \pm 14.5$ & $30.9 \pm 24.4$ & $37.5 \pm 24.7$ \\
\hline Cortisol $(\mathrm{ng} / \mathrm{ml})$ & $1.46 \pm 0.59$ & $1.27 \pm 0.76$ & $1.34 \pm 0.72$ & $1.32 \pm 0.86$ & $1.50 \pm 0.59$ & $1.40 \pm 0.42$ & $1.27 \pm 0.62$ & $1.20 \pm 0.51$ \\
\hline
\end{tabular}


Analyses of salivary responses showed main effects of phase for alpha amylase $(F(3,12)=6.3, p<0.01)$ but not for cortisol $(F(3,12)=0.7$, ns). Main effects of protocol and the interaction phase $\mathrm{x}$ protocol were not significant for alpha amylase and cortisol (Table 2).

No differences were observed between the STAI $(42.2 \pm 4.4$ vs $42.1 \pm 4.4)$, and ADS $(13.9 \pm 4.0$ vs $14.1 \pm 3.3)$ scores in HUT and HUT preceded by MA. Similarly, PSS at beginning was $1.6 \pm 0.8$ vs $2.1 \pm 1.0$ and PSS during was $2.5 \pm 0.7$ vs $3.5 \pm 1.1)$ in HUT and HUT preceded by MA.

\section{Discussion}

Application of mental challenge before orthostatic challenge did not affect the orthostatic cardiovascular and hormonal responses. While central drive induced by mental challenge adds to physiologically mediated cardiovascular reflexes and affects orthostatic responses, this effect seems to be absent when additional mental loading is applied before orthostatic challenge.

When comparing the two protocols, there were no differences in heart rate and cardiac output changes. Blood pressure appears to be the primarily regulated variable during mental stress [21], as the increase in blood pressure during mental arousal is due mainly to increases in cardiac output but with an appropriate decrease in vascular resistance. Our results are in agreement with this observation, and similar to results obtained in another study using mental stressors [22].

HUT results after MA, however, were not different when compared to those of HUT alone. This contrasts with significantly different responses when mental challenge occurs simultaneously with orthostatic challenge [30], which could have several explanations. Firstly, mechanisms of cardiovascular regulation have been reported to be different in orthostatic and mental stress: Cardiopulmonary baroreceptor unloading due to central hypovolemia occurs with orthostatic stress while an increase in central command and arterial baroreceptor loading is noticed under mental stress [23]. While during the combination of the stressors, the observed increase in heart rate is attributed to an increase in arterial baroreceptor loading, the extent of loading may be different when the two stressors are applied separately. Secondly, stressor intervals may also play a role. For example, shorter inter-stressor intervals or exposure to a subsequent stronger stressors leads to reduced hormonal responses [2,3]. In our study, mental challenge was followed immediately by orthostatic challenge. However, the subjects perceived both stresses as similar. Thirdly, it is possible that the maximal effect of mental challenge was over by the time the orthostatic loading occurred, since a time dependent decrease in the magnitude of hemodynamic responses to mental stress has been previously observed [24].

Power spectral analysis of heart rate variability [15] showed similar changes in heart rate variability and baroreceptor sensitivity in both protocols: The high frequency component of RRI intervals decreased while the low frequency component and consequently, the LF/HF ratio (vasovagal balance/quotient: VQ) increased.
Baroreceptor sensitivity decreased as well, suggesting an increased autonomic reactivity, which is in agreement with earlier reports [25].

Baroreflex function can be modulated by behavior/ mental challenges at relay sites in the medulla, pons and hypothalamus [26]. Furthermore, mental arithmetic modifies the normal input to the baroreceptors [27]. In our study, in which the mental challenge preceded the orthostatic loading, it appears that the additive influences on the orthostatic responses are not present when mental challenge is applied separately from orthostatic challenge.

No differences between the two protocols were observed for saliva cortisol. This is consistent with the observation that humoral activation, in comparison to neurovascular effects, shows delayed responses, even more so when the measurement is in saliva rather than blood [13]. Alternatively, the mental challenge used may be considered a weak stress stimulus, which was not intensive enough to trigger the activity of the HPA axis [28].

The activity of alpha amylase showed differences during the experiments irrespective of the protocols. Alpha amylase has been shown to increase during physical stress; reported responses to mental stress, however, are inconsistent [17]. The possibility that the increased amylase could be due to production of saliva supporting the speech task that was part of the mental challenge needs to be further explored.

Differences in stress perception during- and beginning of the challenges were significant. However, when comparing differences in perceived stress between the two protocols, stress perception was not statistically different. Similarly, measures of emotional assessment were also not different between the protocols.

Changes in respiratory frequency affect heart rate variability [15]. Within the two protocols, there was no difference in responses of end tidal $\mathrm{CO}_{2}$ and respiratory frequency. Therefore, the hemodynamic responses observed in normal healthy subjects, who are breathing normally, could be attributed to the stressors per se and the variabilites in heart rate and blood pressure to baroreflex modulation [29].

\section{Limitations}

Blood pressure changes reported here may not only reflect modulation of the autonomic system but vascular reactivity arising due to autonomic stimulation [25]. Moreover, spectral indices do not provide a real measure of nerve activities but only are markers of their overall interaction with target function [15]. Finally, we could not discriminate effects of mental arithmetic from the known effects of just talking on blood pressure.

\section{Conclusions and clinical applications}

Using additional mental challenge before orthostatic loading, we conclude that the orthostatic cardiovascular responses were similar to those observed when orthostatic challenge was applied alone. These results imply that mental challenge preceding orthostatic loading may 
not improve blood pressure response in subjects, upon standing up.

In investigating the effects of mental challenge on orthostatic tolerance, it is important to apply the mental stress together with rather than before- orthostatic load. We propose that in studies in which mental challenge is used as a possible tool to improve orthostatic tolerance, the mental challenge should be applied for the last few minutes preceding orthostatic limit (presyncopal state). Let us take two subjects (A and B) whose orthostatic tolerance times are 15 and $30 \mathrm{~min}$, respectively. It would benefit subject A significantly if mental challenge is applied after the first $5 \mathrm{~min}(5-15 \mathrm{~min})$ of orthostatic loading while $B$ would benefit more if the mental challenge is applied during the last $10 \mathrm{~min}$ (from 20 to $30 \mathrm{~min}$ ) of orthostatic loading. It is possible that the maximal effects of mental challenge on orthostatic cardiovascular responses might be over in subject B if it is applied like in subject A (i.e. between 5 and $15 \mathrm{~min}$ ).

Future research should identify the duration a stressor requires, when applied singly or in combination, to elicit peak cardiovascular responses (that is, time course of responses). This is particularly important as longer stress applications result in habituation and adaptation of responses.

\section{Conflict of interest}

None declared.

\section{Acknowledgments}

We wish to thank Dr Andreas Roessler, Dr Erik Grasser and $\mathrm{Mr}$ Andreas Jantscher, Medical University of Graz, for their excellent technical assistance. We also wish to thank the subjects for their patience and time.

Funding

This study was supported by the Austrian Research Promotion Agency (FFG project 817086 'Orthocap').

\section{References}

[1] J.R. Carter, N.T. Kupiers, C.A. Ray, Neurovascular responses to mental stress, J. Physiol.-London 564 (2005) 321-327.

[2] J. Graessler, R. Kvetnansky, D. Jezova, M. Dobrakovova, G.R. Vanloon, Prior immobilization stress alters adrenal-hormone responses to hemorrhage in rats, Am. J. Physiol. 257 (1989) R661-R667.

[3] O. Marti, R. Andres, A. Armario, Defective ACTH response to stress in previously stressed rats: dependence on glucocorticoid status, Am. J. Physiol.-Regul. Integrat. Comp. Physiol. 277 (1999) R869-R877.

[4] G. Aguilera, Regulation of pituitary acth-secretion during chronic stress, Front. Neuroendocrinol. 15 (1994) 321-350.

[5] R. McCarty, K. Horwatt, M. Konarska, Chronic stress and sympathetic-adrenal medullary responsiveness, Soc. Sci. Med. 26 (1988) 334-341.

[6] N. Goswami, J.A. Loeppky, H. Hinghofer-Szalkay, LBNP: past protocols and technical considerations for experimental design, Aviat. Space Environ. Med. 79 (2008) 459-471.

[7] V.L. Cooper, R. Hainsworth, Effects of dietary salt on orthostatic tolerance, blood pressure and baroreceptor sensitivity in patients with syncope, Clin. Autonomic Res. 12 (2002) 236-241.
[8] P.M. Stevens, Cardiovascular dynamics during orthostasis and influence of intravascular instrumentation, Am. J. Cardiol. 17 (1966) 211-218.

[9] N. Goswami, A. Roessler, H.K. Lackner, D. Schneditz, E. Grasser, H. Hinghofer-Szalkay, Heart rate and stroke volume response patterns to augmented orthostatic stress, Clin. Autonomic Res. 19 (2009) 157-165.

[10] L. Laux, P. Glanzmann, P. Schaffner, C.D. Spielberger, Das State-TraitAngstinventar, Beltz, Weinheim, 1981.

[11] M. Hautzinger, M. Bailer, Allgemeine Depressions Skala, Beltz, Weinheim, 1993

[12] J. Fortin, W. Habenbacher, A. Heller, A. Hacker, R. Gruellenberger, J. Innerhofer, H. Passath, C. Wagner, G. Haitchi, D. Flotzinger, R. Pacher, P. Wach, Non-invasive beat-to-beat cardiac output monitoring by an improved method of transthoracic bioimpedance measurement, Comput. Biol. Med. 36 (2006) 1185-1203.

[13] M.J. Hilz, M. Dutsch, Quantitative studies of autonomic function, Muscle Nerve 33 (2006) 6-20.

[14] H.M. Stauss, Heart rate variability, Am. J. Physiol.-Regul. Integrat. Comp. Physiol. 285 (2003) R927-R931.

[15] M. Pagani, G. Mazzuero, A. Ferrari, D. Liberati, S. Cerutti, D. Vaitl, L. Tavazzi, A. Malliani, Sympathovagal interaction during menta stress-a study using spectral-analysis of heart-rate-variability in healthy control subjects and patients with a prior myocardialinfarction, Circulation 83 (1991) 43-51.

[16] J.P. Saul, R.D. Berger, P. Albrecht, S.P. Stein, M.H. Chen, R.J. Cohen, Transfer-function analysis of the circulation-unique insights into Cardiovascular regulation, Am. J. Physiol. 261 (1991) H1231-H1245.

[17] U.M. Nater, N. Rohleder, J. Gaab, S. Berger, A. Jud, C. Kirschbaum, U. Ehlert, Human salivary alpha-amylase reactivity in a psychosocial stress paradigm, Int. J. Psychophysiol. 55 (2005) 333-342.

[18] N. Goswami, E. Grasser A. Roessler, D. Schneditz, H. HinghoferSzalkay, The cardiovascular response to lower body negative pressure in humans depends on seal location, Physiol. Res. 58 (2009) 311-318.

[19] E.K. Grasser, N. Goswami, A. Rossler, K. Vreckoc, H. HinghoferSzalkay, Hemodynamic and neurohormonal responses to extreme orthostatic stress in physically fit young adults, Acta Astronaut. 64 (2009) 688-696

[20] H.G. Hinghofer-Szalkay, N. Goswami, A. Rossler, E. Grasser, D. Schneditz, Reactive hyperemia in the human liver, Am. J. Physiol. Gastrointest. Liver Physiol. 295 (2008) G332-G337.

[21] S. Julius, The blood-pressure seeking properties of the central nervous system, J. Hypertension 6 (1988) 177-185.

[22] M. Lindqvist, A. Melcher, P. Hjemdahl, Hemodynamic and sympathoadrenal responses to mental stress during nitric oxide synthesis inhibition, Am. J. Physiol.-Heart Circ. Physiol. 287 (2004) H2309-H2315.

[23] C.A. Sweene, M. Bootsma, H.H. Van Bolhuis, Different autonomic responses to orthostatic and to mental stress in young normals, Homeostasis 36 (1995) 287-292.

[24] I.D. Sant'Anna, E.B. De Sousa, A.V. De Moraes, D.L. Loures, E.T. Mesquita, A.C.L. Da Nobrega, Cardiac function during mental stress: cholinergic modulation with pyridostigmine in healthy subjects, Clin. Sci. 105 (2003) 161-165.

[25] A. Radaelli, L. Bernardi, F Valle, S. Leuzzi, F. Salvucci, L. Pedrotti, E. Marchesi, G. Finardi, P. Sleight, Cardiovascular autonomic modulation in essential-hypertension-effect of tilting, Hypertension 24 (1994) 556-563.

[26] R.B. Stephenson, Modification of reflex regulation of blood-pressure by behavior, Ann. Rev. Physiol. 46 (1984) 133-142.

[27] I.B. Goldstein, D. Shapiro, Cardiovascular-responses to mental arithmetic and handgrip during different conditions of postural change, Psychophysiology 25 (1988) 127-136.

[28] D. Jezova, N. Hlavacova, Endocrine factors in stress and psychiatric disorders focus on anxiety and salivary steroids, Stress, Neurotransmitters Horm.: Neuroendocrine and Genet. Mech. 1148 (2008) 495-503.

[29] L. Bernardi, S. Leuzzi, A. Radaelli, C. Passino, J.A. Johnston, P. Sleight, Low-frequency spontaneous fluctuations of $\mathrm{r}-\mathrm{r}$ interval and bloodpressure in conscious humans-a baroreceptor or central phenomenon, Clin. Sci. 87 (1994) 649-654.

[30] N. Goswami, H.K. Lackner, I. Papousek, D. Jezova, J.P. Montani, $H$. Hinghofer-Szalkay, Interaction of mental and orthostatic stressors, Acta Astronautica (2009), this issue. 